\title{
CONCEPTUALISATION ONTOLOGIQUE DE LA REPRÉSENTATION DU COMBAT
}

\author{
Sylvain Rheault, Université de Regina
}

\section{Résumé analytique}

En adoptant une perspective existentielle, on peut tenter d'expliquer la représentation du combat au moyen de concepts comme les positions de SOI et de L'AUTRE ainsi que les statuts de l'ÊTRE et de la CHOSE, qui, une fois combinés, définissent des domaines ontologiques. Le combat devient alors, conceptuellement, l'action de forcer une conscience à passer d'un domaine à un autre. On observe qu'en modifiant l'intensité des positions (polarisation), des statuts (hiérarchisation) et des actions (dosage), on peut expliquer les variations possibles des représentations du combat. Il restera à valider la pertinence de ces concepts en multipliant les analyses.

Henri Barbusse relate I'horreur de la guerre des tranchées dans Le Feu. Malraux transforme le soulèvement des communistes de Shanghaï en épopée dans La Condition Humaine. Simone de Beauvoir, dans Le Deuxième Sexe, théorise la lutte d'émancipation des femmes. Antonine Maillet, dans Les Cordes-de-Bois, oppose ceux de la butte à ceux du village. Ces oeuvres, aussi diverses qu'elles puissent être, traitent du combat. Mais qu'est-ce que le combat, quand il s'insère dans une trame littéraire? En pastichant Mallarmé, on pourrait dire que le combat est l'absent de tout champ de bataille. L'esprit humain, pour appréhender virtuellement le combat, doit procéder à un décodage au moyen des principes ontologiques qui régissent sa perception de la réalité. Simone Weil, dans Les Sources grecques, a proposé que "la force devant quoi la chair des hommes se rétracte" soit le sujet même de L'Iliade et cette démarche s'est avérée à notre avis des plus productives. Nous avons pensé étoffer cette démarche initiale avec les théories élémentaires de l'existentialisme, plus particulièrement celles élaborées par Simone de Beauvoir dans Pyrrhus et Cinéas. Il faut cependant prendre garde que théoriser au moyen de concepts abstraits puisse amener à adopter une approche étroite, qui ne sera peut-être valable que pour l'étude de textes occidentaux. Le but du présent article consiste à élaborer une schématisation qui permettra de mieux comprendre les enjeux de la représentation du combat en se gardant toutefois de prétendre exposer toute la vérité sur la question. Exposons maintenant les concepts utiles en les illustrant au moyen de quelques exemples tirés de la littérature du XXe siècle et en explorer les variations possibles.

Premier concept: les positions de SOI et de L'AUTRE

À la suite de Descartes, établissons que toute relation au monde et à ses représentations part de la conscience d'exister: cogito ergo sum. De là, on peut élaborer le premier principe de la représentation du combat, c'est-à-dire que l'esprit humain distingue deux positions ontologiques: SOI et L'AUTRE. Sans refaire I'histoire de la pensée essentialiste, il suffit de savoir que ce sont des philosophes comme Jaspers qui en sont venus à établir ces 
deux positions (Foulquié, 1966, 111). Ces positions ontologiques (SOI et L'AUTRE), il est possible d'en faire varier le degré de "polarisation". Il s'agit d'exacerber ou non les distinctions entre la première entité et la seconde? Hitler, dans Mein Kampf, contraste sans cesse les qualités de l'Aryen aux défauts du Juif. Malraux ne montre que des Républicains dans L'Espoir. Quoique subtile, cette manière de procéder par omission, n'en reste pas moins une façon de créer de la distance, de polariser les groupes ennemis. En revanche, Antonine Maillet, dans Les Cordesde-bois, non seulement consacre la même longueur de texte à décrire les agissements de ceux de la colline et de ceux du village, en plus, elle les rassemble souvent en des lieux communs, comme à l'église, pour finalement enrôler tous les personnages sous la bannière de la culture acadienne. Maillet "dépolarise" les camps.

Deuxième concept: les statuts de L'ÊTRE et de la CHOSE

La culture occidentale, essentiellement d'inspiration spiritualiste, en est venue à établir une distinction nette entre les êtres et les choses dans l'univers environnant. Un ÊTRE, c'est une créature vivante, mais c'est surtout un être humain. L'ÊTRE, formé de matière comme la chose, s'en distinguerait cependant par un principe considéré comme intrinsèque et qu'on ne retrouverait pas dans la CHOSE. On peut nommer ce caractère distinctif "âme" (anima en latin) ou esprit et l'envisager conceptuellement comme une conscience dans une coquille matérielle. Sartre avait fondé son système philosophique sur les pôles de l'être et du néant. En œ sens, on peut considérer la CHOSE et le néant sartrien comme équivalent. En anglais, le rapprochement étymologique est frappant: Thing et No-thing. Le fait de disposer d'une conscience donnerait à l'ÊTRE certains droits dont la chose ne disposerait pas. Essentiellement, il y a la liberté, c'est-à-dire le fait de ne pouvoir être possédé comme une CHOSE, et puis l'accès à la propriété, c'est-à-dire le fait de pouvoir posséder des CHOSES. La déclaration universelle des droits de l'homme reconnaît justement aux individus "le droit à la vie, à la liberté et à la sureté de sa personne" mais aussi que "toute personne, aussi bien seule qu'en collectivité, a droit à la propriété". La CHOSE, au contraire, n'a aucun droit et sa valeur marchande dépend entièrement de son potentiel à susciter le désir. En contraste, l'approche animiste n'établit pas de distinctions entre les êtres et les choses puisque toute manifestation de la nature signale la présence d'un esprit. Tout est ÊTRE. Au contraire, les concepts que nous proposons trahissent une enmntalité essentiellement "occidentaliste". De la partition des entités en statut ontologique (ÊTRE et CHOSE), on peut extrapoler des variations de hiérarchisation. Déjà Diderot, dans Le Rêve de d'Alembert, ordonnait les minéraux, les plantes, les animaux et les hommes en fonction de leur degré de conscience. La théorie de l'évolution de Darwin a souvent été invoquée pour renforcer des opinions racistes. Dans Tintin au Congo, par Hergé, on présente les Africains comme des enfants que le colonisateur doit prendre en main et éduquer. Et il ne faut pas oublier de parler du cas des femmes, reléguées à un statut d'infériorité, même encore aujourd'hui. 
Élizabeth Badinter dénonce d'ailleurs toutes les formes de hiérarchie:

Comme s'ils [les hommes] préféraient la hiérarchie, même au prix de la guerre, à la paix perçue comme source de chaos, de désordre et d'indifférenciation. L'égalité des sexes risque de provoquer la comparaison et de changer les femmes soumises, enfermées dans leur altérité, en concurrentes (Badinter, 189)

Il est intéressant de constater que Badinter semble prédire que l'abolition de la hiérarchisation (femmes soumises) risque de sombrer dans la polarisation (concurrentes).

Troisième concept: quatre domaines ontologiques

En croisant les concepts de positions et de statuts, nous pouvons envisager quatre domaines ontologiques:

$\begin{array}{ll}\text { ÊTRE-SOI } & \text { ÊTRE-AUTRE } \\ \text { CHOSE-SOI } & \text { CHOSE-AUTRE }\end{array}$

L'ÊTRE-SOI définit le domaine de la conscience de soi en tant que point de référence absolu au monde environnant. L'ÊTRE-AUTRE définit le domaine de la conscience de L'AUTRE, impénétrable autrement qu'en recourant à une approche de l'autre conscience, comme on le verra plus loin. Le domaine de la CHOSE-SOI regroupe I'ensemble des possessions matérielles et intellectuelles de I'ÊTRE-SOI. Il s'agit en fait d'une extension de la sphère de I'ÊTRESOI à I'univers matériel. Le domaine de la CHOSE-AUTRE, enfin, regroupe les innombrables possessions matérielles et intellectuelles qui n'appartiennent pas à I'ÊTRE-SOI, qu'il s'agisse d'objets sans propriétaire, comme les pierres du désert, ou d'objets possédés par I'ÊTRE-AUTRE. Observons ce qui arrive quand on agit pour connecter un domaine avec un autre, mais surtout ce qui arrive quand une action fait passer une entité d'un domaine dans un autre.

Quatrième concept: la tolérance et les actions de l'approche et de la métamorphose

Lorsqu'elle est mise en présence d'une autre entité ontologique, I'ÊTRE-SOI peut soit n'envisager aucune action, et on parlera alors de tolérance envers les entités AUTRES, soit travailler à modifier l'état des choses au moyen d'une action. L'action peut être une approche, qui respecte les domaines ontologiques établis (on parlera de communication, ou même de communion), ou, en faisant fi des domaines ontologiques convenus, un viol de l'entité AUTRE qui se voit forcé de passer d'un domaine ontologique à un autre. Le terme qui convient le mieux conceptuellement pour décrire ce passage est celui de "métamorphose", soit le passage d'une forme à une autre. Ce terme apparemment neutre cache des actes répréhensibles, comme des vols, ou des faits insoutenables, comme des meurtres ou des viols. La mise en oeuvre d'une action ontologique implique trois moments:

http://www.brocku.ca/cfra/voixplurielles06-01/index.html 
1 - La situation initiale.

2 - La mise en oeuvre d'une action.

3 - Le résultat de l'action (réussite ou échec).

La mise en oeuvre d'une action peut aboutir soit à une réussite, soit à un échec, et ce dernier résultat entraîne théoriquement un retour à la situation initiale, ou statu quo. Voilà déjà une aberration dans notre conceptualisation. Qu'une action ratée soit sans conséquences aucunes relève de l'utopie. Il y aura toujours des séquelles résiduelles en réalité. Ainsi, même si une tentative de viol avorte, la victime, et parfois l'agresseur, s'en trouveront profondément affectés. Notons que même dans les cas où il n'y aurait pas de contre-action consciente de la part de l'entité métamorphosée, la mise en oeuvre d'une action peut aboutir malgré tout à un échec. C'est que toute entité ontologique, ÊTRE ou CHOSE, dispose d'une capacité intrinsèque de résistance à la métamorphose, qu'elle soit active ou passive. Par exemple, si, en se promenant dans les bois, Paul aperçoit une belle grande pierre qui ornerait bien son jardin, la tentative d'en faire sa possession pourrait se voir frustrée par la masse intransportable de la pierre ou par son adhérence au sol. De même, imaginons un SOI humaniste qui s'appliquerait à "libérer" un AUTRE maintenu à l'état d'esclavage. La tentative de I'humaniste pourrait échouer si cet AUTRE refuse qu'on apporte des changements à sa condition. C'est que l'action métamorphosante n'avait sans doute pas assez de momentum pour vaincre l'inertie ontologique de ce qui devait être métamorphosé.

La mise en oeuvre de l'action peut faire l'objet de variations en intensité. Le dosage dans l'application de la métamorphose oscillera entre un usage adéquat et un usage excessif. Or, si les forces policières sont entraînées à utiliser un minimum de force, en revanche, ceux qui utilisent trop de force apparaîtront comme des criminels. Dans Harry Potter and the Order of the Phoenix, les sorciers affiliés à Voldemort envoient des sorts qui tuent(Aveda Kadavra), alors que leurs opposants se contentent de sorts qui désarment ou immobilisent. Au cours de son expérience de la guerre, le jeune Ferdinand, dans Voyage au bout de la nuit, en vient à se plaindre que les horreurs du combat, "c'est trop". Les terroristes, dans Les Justes de Camus, discutent avec acharnement s'il convient ou non d'assassiner les petits-enfants du Grand-Duc qui voyagent avec ce dernier dans la calèche. C'est qu'il y a des limites à ne pas dépasser. La règle d'or, ici, consiste pour le héros à ne jamais utiliser plus de moyens pour métamorphoser que son adversaire. Malgré les limites imposées par les attentes morales du public, il convient parfois de dramatiser les effets de la métamorphose à des fins de divertissements. La rapidité et l'ampleur d'une transformation constituent les ingrédients d'un bon spectacle. Le grand guignol y allait gaiement dans une surenchère de sang et de dépeçage. Malraux obtient des effets terrifiants dans les dernières pages de La Condition humaine en décrivant les angoisses des révolutionnaires capturés qui envisagent le sort qui les attend: se faire enfourner vivant dans la chaudière d'une locomotive à vapeur. Métaphoriquement, le texte suggère qu'ils seront transformés en coup de sifflet. Chez Colette, en contrepartie, les conflits sont lents à se développer. Dans La 
Chatte, le meurtre de l'animal n'est pas particulièrement spectaculaire. Ce choix permet au texte de développer le conflit interne qui consume la jeune femme.

Conclusion

D'un point de vue existentiel, on peut définir le combat lorsqu'une conscience (SOI ou L'AUTRE) opère une métamorphose, c'est-à-dire faire passer une entité d'un domaine ontologique à un autre, sans le consentement de l'autre conscience. La réussite de la tentative de métamorphose donne au vainqueur le droit de posséder le vaincu comme on possède une CHOSE. D'où la manie choquante des trophées, symboles de chosifications réussies: scalp, têtes réduites, oreilles coupées, pillage, encoches sur la crosse d'une arme, etc. Les métaphores chosifiantes émaillent entre autres les textes de Colette où se développe un combat latent entre certains personnages. Ainsi, dans Claudine à Paris, Claudine déclare "Au point de vue moral, Luce n'existe pas. Je la considère du point de vue physique." (Colette 53)Cette descente du statut d'ÊTRE à celui de CHOSE inspire de nombreuses images dans les récits de guerre, comme le remarque Fussell dans ses analyses des textes de la Grande Guerre:

For the poet Charles Sorley the transformation of man into corpse is a three-part action. First man: then, when hit, animal, writhing and thrashing in articulate agony or making horrible snoring noises; then "thing". (Fussell 126)

Bref, si la conceptualisation de la représentation du combat n'est pas sans inconvénients, les possibilités semblent malgré tout assez prometteuses. Il restera à poursuivre la validation des concepts sur des textes aussi divers que possible.

\section{Corpus}

Barbusse, Henri. Le Feu. 1916. Paris: Flammarion, 1965.

Camus, Albert. Les Justes. 1949. Paris: Gallimard, 1997.

Céline, Louis-Ferdinand. Voyage au bout de la nuit. 1932. Paris: Gallimard, 1996.

Collette et Willy. Claudine à Paris. 1901. Paris: Albin Michel, 1980.

Colette. La Chatte. 1933. Paris: Hachette, 1960.

Diderot, Denis. Le Rêve de d'Alembert. 1769. Paris: Éditions sociales, 1971.

Hergé. Tintin au Congo. 1931. Bruxelles: Casterman, 1967.

Hitler, Adolf. Mein Kampf. 1925. Boston: Houghton Mifflin Company, 2001.

http://www.brocku.ca/cfra/voixplurielles06-01/index.html 
Maillet, Antonine. Les Cordes-de-Bois. Ottawa: Leméac, 1977.

Malraux, André. La Condition humaine. 1933. Paris: Gallimard, 1996.

---. L'Espoir. 1937. Paris: Gallimard, 1996.

Rowling, Joanne K. Harry Potter and the Order of the Phoenix. Vancouver: Raincoast Books, 2005.

\section{Bibliographie}

Badinter, Elisabeth. L'Un est l'autre. Paris: Odile Jacob, 1986.

Beauvoir, Simone de. Pyrrhus et Cinéas. Paris: Gallimard, 1944.

---. Le Deuxième Sexe. Paris: Gallimard, 1949.

Goyet, Florence. Penser sans concepts : fonction de l'épopée guerrière. Paris: Honoré Champion, 2006.

Foulquié, Paul. L'Existentialisme. Paris: Presses universitaires de France, 1966.

Fussel, Paul. The Great War and Modern Memory. 1975. Oxford: Oxford University Press, 1977.

Weil, Simone. Les Sources grecques. Paris: Gallimard, 1953.

http://www.brocku.ca/cfra/voixplurielles06-01/index.html 